\title{
Rational harmonic balance based method for conservative nonlinear oscillators: Application to the Duffing equation
}

\author{
A. Beléndez, E. Gimeno, T. Beléndez and A. Hernández \\ Departamento de Física, Ingeniería de Sistemas y Teoría de la Señal. \\ Universidad de Alicante. Apartado 99. E-03080 Alicante. SPAIN
}

E-mail: a.belendez@ua.es

Corresponding author: A. Beléndez (Phone: +34-6-5903651, Fax: +34-6-5903464)

\begin{abstract}
A second-order modified rational harmonic balance method is used approximately solve the nonlinear differential equation that governs the oscillations of a conservative autonomous system with one degree of freedom. The Duffing oscillator is analyze to illustrate the usefulness and effectiveness of the proposed technique. We find that this method works very well for this oscillator, and excellent agreement of the approximate frequencies with the exact one has been demonstrated and discussed. For the second-order approximation we have shown that the relative error in the analytical approximate frequency is as low as $0.0055 \%$ when $A$ tends to infinity. We also compared the Fourier series expansions of the analytical approximate solution and the exact one. This has allowed us to compare the coefficients for the different harmonic terms in these solutions.
\end{abstract}

Keywords: Nonlinear oscillators; Approximate solutions; Rational harmonic balance method.

\section{Introduction}

Nonlinear oscillator models have been widely used in many areas not only in physics and engineering, but also they are of significant importance in other fields. Mechanical oscillatory systems are often governed by non-linear differential equations. It is well known that a nonlinear equation of this type is often linearized by retaining the first term of the Taylor series expansion of the restoring force in the neighbourhood of the equilibrium point. This procedure yields acceptable results for many cases, but is unable to show the amplitude dependence of the oscillation period $[1,2]$. There are a large variety of approximate methods commonly used for solving nonlinear oscillatory systems including perturbations [1-4], variational [5, 6], variational iteration [7-10], homotopy perturbation [11-16] or harmonic balance [2, 17-24] methods, etc. Surveys of the literature with numerous references and useful bibliography and a review of these methods can be found in detail in [16] and [25]. In this paper we apply a modified generalized, rational harmonic balance method to obtain analytic approximate solutions for conservative nonlinear oscillators and, in particular, the Duffing equation is analyzed and discussed. In this method the approximate solution obtained approximates all of the harmonics in the exact solution [26, 27], whereas the usual harmonic balance techniques provide an approximation to only the lowest harmonic components. For most cases, the application of the rational harmonic balance method leads to very complicated sets of algebraic equations with very complex nonlinearity that have to be solved even for the second-order approximation. In an attempt to provide better solution methodology a modification in this technique is proposed for constructing the secondorder analytical approximate solution. Comparison with the exact solution as well as with the results obtained using the standard harmonic balance method is also included. The most interesting features of the proposed method are its simplicity and its excellent accuracy in a wide range of values of oscillation amplitude. 


\section{Formulation and solution method}

Consider the simplest nonlinear conservative autonomous system encountered in the theory of oscillations with one degree of freedom, whose motion is governed by the following dimensionless second order differential equation

$$
\frac{\mathrm{d}^{2} x}{\mathrm{~d} t^{2}}+f(x)=0, \quad x(0)=A, \quad \frac{\mathrm{d} x}{\mathrm{~d} t}(0)=0
$$

where the nonlinear restoring-force function $f(x)$ is odd, i.e. $f(-x)=-f(-x)$. The motion is assumed to be periodic and the problem is to determine the angular frequency of oscillation and corresponding solution as a function of the system parameters and the amplitude $A$.

To solve Eq. (1) by the modified rational harmonic balance method, a new independent variable $\tau=\omega t$ is introduced, so Eq. (1) can be rewritten as

$$
\omega^{2} \frac{\mathrm{d}^{2} x(\tau)}{\mathrm{d} \tau^{2}}+f(x(\tau))=0, \quad x(0)=A, \quad \frac{\mathrm{d} x}{\mathrm{~d} \tau}(0)=0
$$

The new variable is chosen in such a way that the solution of Eq. (2) is a periodic function of $\tau$ of period $2 \pi$. In order to determine an analytical approximate solution to Eq. (2) we use a rational form given by the following expression [1]

$$
x_{2}(\tau)=\frac{A\left(1+B_{2}\right) \cos \tau}{1+B_{2} \cos 2 \tau}
$$

where it satisfies that $x_{2}(0)=A$ and $\left|B_{2}\right|<<1$. Substituting Eq. (3) into Eq. (2) leads to

$$
-\omega^{2} A\left(1+B_{2}\right)\left\lfloor\frac{\cos \tau}{1+B_{2} \cos 2 \tau}-\frac{4 B_{2} \cos 3 \tau}{\left(1+B_{2} \cos 2 \tau\right)^{2}}-\frac{8 B_{2}^{2} \cos \tau \sin ^{2} 2 \tau}{\left(1+B_{2} \cos 2 \tau\right)^{3}}\right\rfloor+f\left(\frac{A\left(1+B_{2}\right) \cos \tau}{1+B_{2} \cos 2 \tau}\right)=0
$$

which can be written as follows $F\left(A, B_{2}, \omega, \tau\right)=0$. As $\left|B_{2}\right|<<1$ we can do the following Taylor's series expansion

$$
F\left(A, B_{2}, \omega, \tau\right) \approx F_{0}(A, \omega, \tau)+F_{1}(A, \omega, \tau) B_{2}+F_{2}(A, \omega, \tau) B_{2}^{2}
$$

where

$$
\begin{gathered}
F_{0}(A, \omega, \tau)=-A \omega^{2} \cos \tau+G_{0}(A, \tau) \\
F_{1}(A, \omega, \tau)=-\frac{1}{2} \omega^{2} A(\cos \tau-9 \cos 3 \tau)+G_{1}(A, \tau) \\
F_{2}(A, \omega, \tau)=\frac{1}{4} \omega^{2} A(9 \cos 3 \tau-25 \cos 5 \tau)+G_{3}(A, \tau) \\
G_{0}(A, \tau)=f(A \cos \tau) \\
G_{1}(A, \tau)=\frac{1}{2} A(\cos \tau-\cos 3 \tau) f_{x}(A \cos \tau) \\
G_{2}(A, \tau)=\frac{1}{4} A(\cos 5 \tau-\cos 3 \tau) f_{x}(A \cos \tau)+\frac{1}{16} A^{2}(2-\cos 2 \tau-2 \cos 4 \tau+\cos 6 \tau) f_{x x}(A \cos \tau)
\end{gathered}
$$

where the subscript $x$ denotes derivative of $f(x)$ with respect to $x$. Expanding Eq. (5) in a trigonometric series we can write

$$
H^{(1)}\left(A, B_{2}, \omega\right) \cos \tau+H^{(3)}\left(A, B_{2}, \omega\right) \cos 3 \tau+H O H=0
$$


where $\mathrm{HOH}$ stands for higher-order harmonics and

$$
\begin{aligned}
& H^{(1)}\left(A, B_{2}, \omega\right)=f_{0}^{(1)}(A, \omega)+f_{1}^{(1)}(A, \omega) B_{2}+f_{2}^{(1)}(A, \omega) B_{2}^{2} \\
& H^{(3)}\left(A, B_{2}, \omega\right)=f_{0}^{(3)}(A, \omega)+f_{1}^{(3)}(A, \omega) B_{2}+f_{2}^{(3)}(A, \omega) B_{2}^{2}
\end{aligned}
$$

In Eqs. (13) and (14), $f_{m}^{(1)}(A, \omega)$ and $f_{m}^{(3)}(A, \omega)(m=0,1,2)$ are given as follows

$$
\begin{aligned}
f_{m}^{(1)}(A, \omega) & =\frac{4}{\pi} \int_{0}^{\pi / 2} F_{m}(A, \omega, \tau) \cos \tau d \tau \\
f_{m}^{(3)}(A, \omega) & =\frac{4}{\pi} \int_{0}^{\pi / 2} F_{m}(A, \omega, \tau) \cos 3 \tau d \tau
\end{aligned}
$$

Setting the coefficients of $\cos \tau$ and $\cos 3 \tau$ to zero in Eq. (12) it follows that

$$
\begin{aligned}
-A\left(1+\frac{1}{2} B_{2}\right) \omega^{2}+a_{0}(A)+a_{1}(A) B_{2}+a_{2}(A) B_{2}^{2} & =0 \\
\frac{9}{4} A B_{2}\left(2+B_{2}\right) \omega^{2}+b_{0}(A)+b_{1}(A) B_{2}+b_{2}(A) B_{2}^{2} & =0
\end{aligned}
$$

where

$$
\begin{array}{ll}
a_{m}(A)=\frac{4}{\pi} \int_{0}^{\pi / 2} G_{m}(A, \tau) \cos \tau d \tau, & m=0,1,2 \\
b_{m}(A)=\frac{4}{\pi} \int_{0}^{\pi / 2} G_{m}(A, \tau) \cos 3 \tau d \tau, \quad m=0,1,2
\end{array}
$$

Eqs. (17) and (18) allows us to obtain $B_{2}$ and the second order approximate frequency $\omega$ as a function of $A$. Solving Eq. (18) for $\omega$ yields

$$
\omega(A)=\sqrt{\frac{2 a_{0}(A)+2 a_{1}(A) B_{2}(A)+2 a_{2}(A) B_{2}^{2}(A)}{A\left(2+B_{2}(A)\right)}}
$$

and substituting Eq. (21) into Eq. (18) gives the following cubic equation which must be solved to obtain $B_{2}$

where

$$
\alpha_{3}(A) B_{2}^{3}+\alpha_{2}(A) B_{2}^{2}+\alpha_{1}(A) B_{2}+\alpha_{0}(A)=0
$$

$$
\alpha_{0}=b_{0} \quad \alpha_{1}=\frac{9}{2} a_{0}+b_{1} \quad \alpha_{2}=\frac{9}{2} a_{1}+b_{2} \quad \alpha_{3}=\frac{9}{2} a_{2}
$$

As we can see Eq. (22) is cubic and therefore has three solutions. However, the root of interest is real and one for which $\left|B_{2}\right|<<1$. To proceed, assume that such a solution, $B_{2}$, exists and its value is lower than one. A first approximate value $\bar{B}_{2}$ can be determined by neglecting the first two terms in Eq. (22)

$$
\alpha_{1}(A) \bar{B}_{2}+\alpha_{0}(A)=0
$$

Solving this equation gives

$$
\bar{B}_{2}=-\alpha_{0} / \alpha_{1}
$$

To further improve this result, we assume that $B_{2}$ can be written as follows

$$
B_{2}=\bar{B}_{2}+\delta
$$


where $\delta$ is a correction term and $|\delta|<<\left|\bar{B}_{2}\right|$. Substituting Eq. (26) into Eq. (22) and linearizing with respect to the correction term $\delta$ lead to

$$
\alpha_{3} \bar{B}_{2}^{3}\left(1+3 \delta / \bar{B}_{2}\right)+\alpha_{2} \bar{B}_{2}^{2}\left(1+2 \delta / \bar{B}_{2}\right)+\alpha_{1} \bar{B}_{2}\left(1+\delta / \bar{B}_{2}\right)+\alpha_{0}=0
$$

which is a linear equation instead of a cubic equation. Solving Eq. (27) and taking into account Eq. (25), it follows that

$$
B_{2}=-\frac{\alpha_{0}\left(2 \alpha_{0}^{2} \alpha_{3}+\alpha_{1}^{3}-\alpha_{0} \alpha_{1} \alpha_{2}\right)}{\alpha_{1}\left(3 \alpha_{0}^{2} \alpha_{3}+\alpha_{1}^{3}-2 \alpha_{0} \alpha_{1} \alpha_{2}\right)}
$$

\section{Application to the Duffing oscillator}

This oscillator is governed by the following differential equation with initial conditions

$$
\frac{\mathrm{d}^{2} x(t)}{\mathrm{d} t^{2}}+x+\varepsilon x^{3}(t)=0, \quad x(0)=A, \quad \frac{\mathrm{d} x}{\mathrm{~d} t}(0)=0
$$

From Eqs. (19) and (20) it is easy to obtain

$$
\begin{aligned}
& a_{0}=\frac{1}{4} A\left(4+3 \varepsilon A^{2}\right), \quad a_{1}=\frac{1}{4} A\left(2+3 \varepsilon A^{2}\right), \quad a_{2}=\frac{3}{8} \varepsilon A^{3} \\
& b_{0}=\frac{1}{4} \varepsilon A^{3}, \quad b_{1}=-\frac{1}{8} A\left(4+3 \varepsilon A^{2}\right), \quad b_{2}=-\frac{1}{4} A\left(1+3 \varepsilon A^{2}\right)
\end{aligned}
$$

and taking into account Eq. (23)- we can write $B_{2}$ (Eq. (35)) as follows

$$
B_{2}(\lambda)=-\frac{\lambda\left(8192+18176 \lambda+13296 \lambda^{2}+3231 \lambda^{3}\right)}{2(4+3 \lambda)\left(16384+35840 \lambda+25536 \lambda^{2}+5985 \lambda^{3}\right)}
$$

where $\lambda=\varepsilon A^{2}$. The analytical approximate frequency has the following expression

$$
\omega_{\text {RHBM } 2}(\lambda)=\sqrt{\frac{h_{0}+h_{1} \lambda+h_{2} \lambda^{2}+h_{3} \lambda^{3}+h_{4} \lambda^{4}+h_{5} \lambda^{5}+h_{6} \lambda^{6}+h_{7} \lambda^{7}+h_{8} \lambda^{8}+h_{9} \lambda^{9}}{8\left(g_{0}+g_{1} \lambda+g_{2} \lambda^{2}+g_{3} \lambda^{3}+g_{4} \lambda^{4}\right)\left(k_{0}+k_{1} \lambda+k_{2} \lambda^{2}+k_{3} \lambda^{3}+k_{4} \lambda^{4}\right)}}
$$

where

$$
\begin{aligned}
& h_{0}=137438953472 \\
& h_{3}=4472235360256 \\
& h_{6}=1660534023168 \\
& h_{9}=7072335423 \\
& g_{2}=209664 \\
& k_{0}=262144 \\
& k_{3}=388896
\end{aligned}
$$$$
h_{1}=906238099456
$$$$
h_{2}=2642344411136
$$$$
h_{4}=4843127439360
$$$$
h_{5}=3480739848192
$$$$
h_{7}=507162127392
$$$$
h_{8}=90002965320
$$$$
g_{0}=65536
$$$$
g_{1}=192512
$$$$
g_{3}=100548
$$$$
g_{4}=17955
$$$$
k_{1}=761856
$$$$
k_{2}=820480
$$$$
k_{4}=68589
$$

Therefore, the second approximation to the periodic solution of the nonlinear oscillator is given by the following equation

$$
\frac{x_{2}(t)}{A}=\frac{\left(1+B_{2}\right) \cos \omega t}{1+B_{2} \cos 2 \omega t}
$$

which has the following Fourier series expansion 


$$
\begin{gathered}
\frac{x_{2}(t)}{A}=\sum_{n=0}^{\infty} c_{2 n+1} \cos [(2 n+1) \omega t] \\
C_{2 n+1}=\frac{4}{\pi} \int_{0}^{\pi / 2} \frac{\left(1+B_{2}\right) \cos \tau}{1+B_{2} \cos 2 \tau} \cos [(2 n+1) \tau] d \tau
\end{gathered}
$$

As we can see, Eq. (34) gives an expression that approximates all of the harmonics in the exact solution whereas the usual harmonic balancing techniques provide an approximation to only the lowest harmonic components [26-28].

We illustrate the accuracy of the modified approach by comparing the approximate solutions previously obtained with the exact frequency $\omega_{e}$ and other results in the literature. In particular we will consider the solution of Eq. (29) by means of the second- and third-order linearized harmonic balance method [23]. Direct integration of Eq. (29) yields the exact frequency as [1]

$$
\omega_{e}(\lambda)=\frac{\pi}{2} \sqrt{1+\lambda} / K\left(\frac{\lambda}{2(1+\lambda)}\right)
$$

where $K(m)$ is the complete elliptical integral of the first kind. Table 1 shows comparison of the exact frequency in Eq. (37) and the approximate frequency $\omega_{\text {RHBM } 2}$ in Eq. (33). As can be observed, Eq. (33) gives excellent approximate solution for both small and large $\lambda$. In this table the values of $B_{2}$ for each value of $\lambda$ obtained using Eq. (32) have been also included.

Table 1.- Comparison of the approximate frequency $\left(\omega_{R H B M 2}\right)$ with the exact one $\left(\omega_{e}\right)$.

\begin{tabular}{lllll}
\hline$\lambda$ & $B_{2}$ & $\omega_{\text {RHBM } 2}$ & $\omega_{e}$ & Percentage error $(\%)$ \\
\hline-0.95 & 0.254002 & 0.432221 & 0.437570 & $1.2 \%$ \\
-0.9 & 0.191124 & 0.504846 & 0.506353 & $0.30 \%$ \\
-0.5 & 0.0493704 & 0.784551 & 0.784553 & $0.00026 \%$ \\
-0.3 & 0.0239711 & 0.878790 & 0.878790 & $0.0000069 \%$ \\
-0.1 & 0.00673547 & 0.961637 & 0.961637 & $0.000000012 \%$ \\
0.5 & -0.0230452 & 1.17078 & 1.17078 & $0.0000057 \%$ \\
1 & -0.0365864 & 1.31778 & 1.31778 & $0.000057 \%$ \\
5 & -0.0694731 & 2.15045 & 2.15042 & $0.0015 \%$ \\
10 & -0.0783777 & 2.86672 & 2.86664 & $0.0027 \%$ \\
100 & -0.0886590 & 8.53402 & 8.53359 & $0.0051 \%$ \\
$\rightarrow \infty$ & -0.0899749 & $0.847260 \sqrt{\lambda}$ & $0.847213 \sqrt{\lambda}$ & $0.0055 \%$ \\
\hline
\end{tabular}

Wu et al. [23] approximately solved Eq. (1) using an improved harmonic balance method that incorporates salient features of both Newton's method and the harmonic balance method. They achieved the following results for the second approximation order

$$
\omega_{\text {HBM } 2}(\lambda)=\sqrt{\frac{128+192 \lambda+69 \lambda^{2}}{128+96 \lambda}}
$$

and the following result for the third order 


$$
\omega_{\text {HBM } 3}(\lambda)=\sqrt{\frac{r_{0}+r_{1} \lambda+r_{2} \lambda^{2}+r_{3} \lambda^{3}+r_{4} \lambda^{4}+r_{5} \lambda^{5}+r_{6} \lambda^{6}+r_{7} \lambda^{7}+r_{8} \lambda^{8}+r_{9} \lambda^{9}}{16(4+3 \lambda)\left(s_{0}+s_{1} \lambda+s_{2} \lambda^{2}+s_{3} \lambda^{3}+s_{4} \lambda^{4}+s_{5} \lambda^{5}+s_{6} \lambda^{6}+s_{7} \lambda^{7}\right)}}
$$

where

$$
\begin{aligned}
& r_{0}=1099511627776 \\
& r_{3}=37447618527232 \\
& r_{6}=14654232029184 \\
& r_{9}=65856986475 \\
& s_{2}=197199396864 \\
& s_{5}=76934648832
\end{aligned}
$$$$
r_{1}=7352984010752
$$$$
r_{2}=21769041739776
$$$$
r_{4}=41248951894016
$$$$
r_{5}=30171363606528
$$$$
r_{7}=4557352944960
$$$$
r_{8}=823439591472
$$$$
s_{0}=17179869184
$$$$
s_{1}=89120571392
$$$$
s_{3}=241278386176
$$$$
s_{4}=176300392448
$$$$
s_{6}=18565607712
$$$$
s_{7}=1911234801
$$

For small values of $\lambda$ it is possible to do the power series expansions of the exact and approximate angular frequencies. Doing these expansions, the following equations can be obtained

$$
\begin{aligned}
& \omega_{e}(\lambda)=1+\frac{3}{8} \lambda-\frac{21}{256} \lambda^{2}+\frac{81}{2048} \lambda^{3}-\frac{6549}{262144} \lambda^{4}+\frac{37737}{2097152} \lambda^{5}+\ldots \\
& \omega_{\text {RHBM } 2}(\lambda)=1+\frac{3}{8} \lambda-\frac{21}{256} \lambda^{2}+\frac{81}{2048} \lambda^{3}-\frac{6549}{262144} \lambda^{4}+\frac{37754.25}{2097152} \lambda^{5}+\ldots \\
& \omega_{\text {HBM } 2}(\lambda)=1+\frac{3}{8} \lambda-\frac{21}{256} \lambda^{2}+\frac{81}{2048} \lambda^{3}-\frac{6498}{262144} \lambda^{4}+\frac{36666}{2097152} \lambda^{5}+\ldots \\
& \omega_{\text {HвM } 3}(\lambda)=1+\frac{3}{8} \lambda-\frac{21}{256} \lambda^{2}+\frac{81}{2048} \lambda^{3}-\frac{6543}{262144} \lambda^{4}+\frac{37607.25}{2097152} \lambda^{5}+\ldots
\end{aligned}
$$

As can be seen, for small values of $A$ the frequency obtained using the modified RHBM is better than the other two frequencies. On the other hand, in Eq. (41), the first five terms are the same as the first three terms obtained from the expansion of the exact frequency (Eq. (40)), whereas the sixth term of the expansion of the exact frequency is $\frac{37737}{2097152}$ compared with $\frac{37754.25}{2097152}$ obtained in this study, that is, the relative error in this term is less than $0.05 \%$. For large values of $\lambda$ it is also possible to do the power series expansions of the exact and approximate angular frequencies and we obtain

$$
\begin{aligned}
& \omega_{e}(\lambda)=\frac{\Gamma^{2}(3 / 4) \sqrt{\lambda}}{\sqrt{\pi}}+\frac{\Gamma^{4}(3 / 4)+4 \Gamma^{2}(3 / 4) \Gamma^{2}(5 / 4)}{8 \sqrt{\pi \lambda} \Gamma^{2}(5 / 4)}+\ldots=0.847213 \sqrt{\lambda}+\frac{0.617172}{\sqrt{\lambda}}+\ldots \\
& \omega_{R H B M 2}(\lambda)=\frac{1}{2} \sqrt{\frac{29104261 \lambda}{10135930}}+\frac{1211141480509}{114315 \sqrt{2949988752197730 \lambda}}+\ldots=0.847260 \sqrt{\lambda}+\frac{0.616853}{\sqrt{\lambda}}+\ldots \\
& \omega_{\text {HBM } 2}(\lambda)=\sqrt{\frac{23 \lambda}{32}}+\frac{25}{6 \sqrt{46 \lambda}}+\ldots=0.847791 \sqrt{\lambda}+\frac{0.614341}{\sqrt{\lambda}}+\ldots \\
& \omega_{\text {HBM } 3}(\lambda)=\frac{5}{4} \sqrt{\frac{125441879 \lambda}{273033543}}+\frac{2181990577378901983}{19112348010 \sqrt{34249840663947297 \lambda}}+\ldots=0.847273 \sqrt{\lambda}+\frac{0.616893}{\sqrt{\lambda}}+\ldots
\end{aligned}
$$

In Eq. (44), $\Gamma(z)$ is the Euler gamma function.

Taking into account Eq. (32), we can also obtain the power series expansion of $B_{2}$ for large values of $\lambda$ and we obtain

$$
B_{2}(\lambda)=-\frac{359}{3990}+\frac{3998}{29925 \lambda}+\ldots=-0.0899749+\frac{0.133601}{\lambda}+\ldots
$$


and then $\lim _{\lambda \rightarrow \infty} B_{2}(\lambda)=-\frac{359}{3990}=-0.0899749$. All series expansions were carried out using MATHEMATICA. Furthermore we have

$$
\begin{gathered}
\lim _{\lambda \rightarrow \infty} \frac{\omega_{R H B M 2}(\lambda)}{\omega_{e}(\lambda)}=\frac{1}{2 \Gamma^{2}(3 / 4)} \sqrt{\frac{29104261 \pi}{10135930}}=1.000055 \\
\lim _{\lambda \rightarrow \infty} \frac{\omega_{\text {HBM } 2}(\lambda)}{\omega_{e}(\lambda)}=\frac{1}{4 \Gamma^{2}(3 / 4)} \sqrt{\frac{23 \pi}{2}}=1.00068 \\
\lim _{\lambda \rightarrow \infty} \frac{\omega_{\text {HBM } 3}(\lambda)}{\omega_{e}(\lambda)}=\frac{5}{4 \Gamma^{2}(3 / 4)} \sqrt{\frac{125441879 \pi}{273033543}}=1.000071
\end{gathered}
$$

Once again, for large values of $A$ the frequency $\omega_{\text {RHBM } 2}$ obtained using the modified RHBM is better than the other two frequencies and its relative error in relation the exact frequency tends to $0.0055 \%$ when $A$ tends to infinity. However the relative errors for $\omega_{\mathrm{HBM} 2}$ and $\omega_{\mathrm{HBM} 3}$ tend to $0.068 \%$ (around 12 times more than the relative error for $\omega_{\text {RHBM } 2}$ ) and $0.0071 \%$ (around 1.3 times more than the relative error for $\left.\omega_{\text {RHBM } 2}\right)$, respectively. In Figure 1 we plotted the relative errors for the approximate frequencies $\omega_{\text {RHBM } 2}, \omega_{\text {HBM } 2}$ and $\omega_{\text {HBM } 3}$. This figure clearly indicates that $\omega_{R H B M 2}$ is more accurate than the other two frequencies and provides excellent approximations to the exact frequency for the whole range of parameter $\lambda>-1$.

For this problem the exact solution is given as follows [1]

$$
\frac{x_{e}(t)}{A}=c n\left[(1+\lambda)^{1 / 2} t ; k\right], \quad k=\frac{\lambda}{2(1+\lambda)}
$$

where $c n$ is the Jacobi elliptic function which has the following Fourier expansion [1]

$$
\frac{x_{e}(t)}{A}=\frac{2 \pi}{\sqrt{k} K(k)} \sum_{n=0}^{\infty} \frac{q^{n+1 / 2}(k)}{1+q^{2 n+1}(k)} \cos \left[(2 n+1) \omega_{e} t\right], \quad q(k)=\exp \left\lfloor-\frac{\pi K\left(k^{\prime}\right)}{K(k)}\right\rfloor
$$

and $k^{\prime}=1-k=\frac{1}{2}[(2+\lambda) /(1+\lambda)]$.

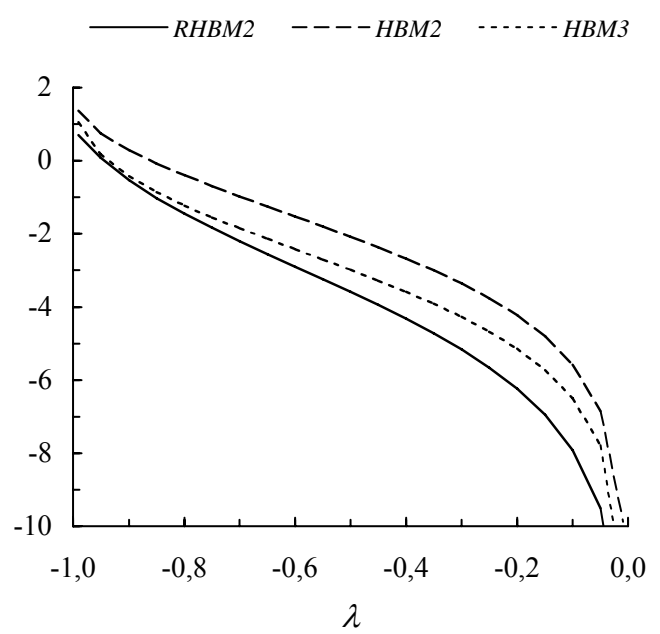

(a)

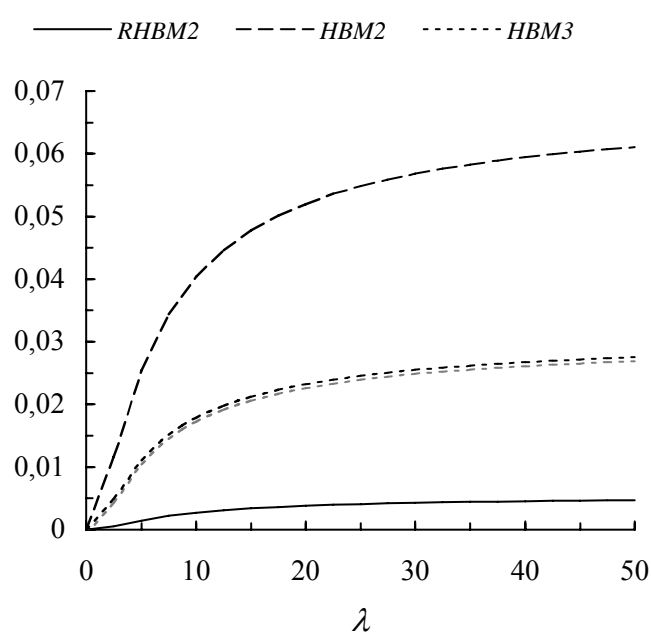

(b)

Fig. 1.- (a) Percentage of error (PE) for approximate frequencies $\omega_{R H B M 2}, \omega_{H B M 2}$ and $\omega_{\text {HBM } 3},-1<\lambda<0$ (decimal logarithm of $P E, \log P E$ ), and (b) $0 \leq \lambda \leq 50$. 
In Table 2 we present the comparison between the first four coefficients of the Fourier series expansions of the exact solution and the second-order approximate solution obtained in this paper. Figure 1 and Table 1 show that the proposed formulas (33) and (34) provide excellent approximations to exact periodic solutions (Eqs. (37) and (42)) for both soft and hard nonlinear oscillators.

At this point it is necessary to answer the following questions: (a) why rational harmonic balance method gives so good results when this is applied to the Duffing oscillator? To answer this question it is necessary to take into account that this oscillator has an exact solution that can be expressed in terms of a Jacobi elliptic function (Eq. (65)). As Mickens pointed out [1], these functions have the feature that they can be written as ratios of series of trigonometric functions. This suggests that approximations to the periodic solutions of Eq. (36) can take the form given in Eq. (5) and that this approximation can give satisfactory results for the Duffing oscillator. We can also add that the rational harmonic balance method will give also satisfactory results for certain oscillation amplitudes intervals for which the behaviour of other nonlinear oscillators is similar to that of a Duffing oscillator with or without a linear term. This is the behaviour, for example, of the Duffing-harmonic oscillator for small values of the oscillation amplitude $[12,18,22]$.

Table 2.- Comparison of the first four coefficients for the Fourier expansions of exact and approximate solutions obtained using the modified rational harmonic balance method considered in this paper. The percentage error is included.

\begin{tabular}{c|cc|cc|cc}
\hline $\begin{array}{c}\text { Fourier } \\
\text { coefficient }\end{array}$ & \multicolumn{2}{|c|}{$\lambda=-0.9$} & \multicolumn{2}{|c}{$\lambda=10$} & \multicolumn{2}{|c}{$\lambda \rightarrow \infty$} \\
\hline$c_{2 n+1}$ & Exact & RHBM & Exact & \multicolumn{1}{c}{ RHBM } & Exact & \multicolumn{1}{c}{ RHBM } \\
\hline$n=0$ & 1.093070 & $1.09645(0.31 \%)$ & 0.960817 & $0.960751(0.0069 \%)$ & 0.955006 & $0.954921(0.0089 \%)$ \\
$n=1$ & -0.102830 & $-0.105754(2.8 \%)$ & 0.0377014 & $0.0377087(0.019 \%)$ & 0.0430545 & $0.0430468(0.018 \%)$ \\
$n=2$ & 0.010784 & $0.010200(5.4 \%)$ & 0.0014256 & $0.0014800(3.8 \%)$ & 0.0018605 & $0.0019405(4.3 \%)$ \\
$n=3$ & -0.001132 & $-0.0009838(13 \%)$ & 0.00005390 & $0.00005809(7.8 \%)$ & 0.00008040 & $0.00008748(8.8 \%)$ \\
\hline
\end{tabular}

\section{Conclusions}

A modified rational harmonic balance method has been applied to obtain analytical approximate solutions for nonlinear problems that are conservative and periodic. This method is based on the substitution of a rational solution - depending of a small parameter $B_{2}$ - into the nonlinear differential equation, Taylor series expansion with respect to the small parameter $B_{2}$, neglect of the terms proportional to $B_{2}^{n}(n \geq 3)$ in the Taylor series expansion, and harmonic balancing. The major conclusion is that this scheme provides excellent approximations to the solution of these nonlinear systems with high accuracy. The Duffing oscillator has been analyzed to illustrate excellent accuracy of the analytical approximate frequencies. The analytical representations obtained using this technique give excellent approximations to the exact solutions for the whole range of values of oscillation amplitudes. For the second order approximation, the relative error of the analytical approximate frequency obtained using the approach considered in this paper is lower than $0.0055 \%$ for $A \in[0, \infty[$. An interesting feature considered in this paper is the comparison between the analytical approximate solutions and the Fourier series expansion of the exact solution. This has allowed us to compare the coefficients for the different harmonics. The results presented in this paper are the best reported for this type of nonlinear oscillator at the present time and they conform the application of this modified rational harmonic balance method for other conservative nonlinear oscillators. 
A. Beléndez, E. Gimeno, T. Beléndez and A. Hernández, "Rational harmonic balance based method for conservative nonlinear oscillators: Application to the Duffing equation", Mechanics Research Communications, Vol. 36, No 6, 728-734 (2009). DOI: 10.1016/j.mechrescom.2009.03.001

\section{Acknolowgements}

This work was supported by the "Generalitat Valenciana" of Spain, under Project GREP/2007/020.

\section{References}

[1] R. E. Mickens, Oscillations in Planar Dynamics Systems (World Scientific, Singapore 1996).

[2] J. H. He, Non-perturbative methods for strongly nonlinear problems (dissertation.de-Verlag im Internet $\mathrm{GmbH}$, Berlin 2006).

[3] A. H. Nayfeh, Problems in Perturbations (Wiley, New York 1985).

[4] P. Amore. A. Raya and F. M. Fernández, "Comparison of alternative improved perturbative methods for nonlinear oscillations", Phys. Lett. A 340, 201-208 (2005).

[5] J. H. He, "Variational approach for nonlinear oscillators", Chaos, Solitons \& Fractals 34, 14301439 (2007).

[6] D. H. Shou, "Variational approach to the nonlinear oscillator of a mass attached to a stretched wire", Phys. Scr. 77, 045006 (2008).

[7] Mo. Miansari, D. D. Ganji and Me. Miansari, "Application of He's variational iteration method to nonlinear heat transfer equations", Phys. Lett. A 372, 779-785 (2008).

[8] J. H. He and X. H. Wu, "Construction of solitary solution and compact on-like solution by variational iteration method", Chaos, Solitons \& Fractals 29, 108-113 (2006).

[9] M. Dehghan and M. Tatari, "The use of He's variational iteration method for solving multipoint boundary value problems", Phys. Scr. 74, 672-676 (2007)

[10] M. Tatari and M. Dehghan, "On the convergence of He's variational iteration method", $J$. Comput. Appl. Math. 207, 121-128 (2007).

[11] J. H. He, "Homotopy perturbation method for bifurcation on nonlinear problems", Int. J. Nonlinear Sci. Numer. Simulat. 6, 207-208 (2005).

[12] A. Beléndez, A. Hernández, T. Beléndez, E. Fernández, M. L. Álvarez and C. Neipp, "Application of He's homotopy perturbation method to the Duffing-harmonic oscillator", Int. J. Non-linear Sci. Numer. Simulat. 8 (1), 79-88 (2007).

[13] A. Beléndez, A. Hernández, T. Beléndez, C. Neipp and A. Márquez, "Application of the homotopy perturbation method to the nonlinear pendulum", Eur. J. Phys. 28, 93-104 (2007)

[14] D. D. Ganji and A. Sadighi, "Application of He's homotopy-perturbation method to nonlinear coupled systems of reaction-diffusion equations", Int. J. Nonlinear Sci. Numer. Simul. 7, 411418 (2006).

[15] T. Özis and A. Yildirim, "A comparative study of He's homotopy perturbation method for determining frequency-amplitude relation of a nonlinear oscillator with discontinuities", Int. $J$. Nonlinear Sci. Numer. Simul. 8, 243-248 (2007).

[16] J. H. He, "Some asymptotic methods for strongly nonlinear equations", Int. J. Mod. Phys. B 20, 1141-1199 (2006).

[17] A. Beléndez and C. Pascual, "Harmonic balance approach to the periodic solutions of the (an)harmonic relativistic oscillator", Phys. Lett. A 371, 291-299 (2007).

[18] H. Hu and J. H. Tang, "Solution of a Duffing-harmonic oscillator by the method of harmonic balance", J. Sound Vib. 294, 637-639 (2006).

[19] C. W. Lim and B. S. Wu, "A new analytical approach to the Duffing-harmonic oscillator", Phys. Lett. A 311, 365-373 (2003).

[20] A. Beléndez, A. Hernández, T. Beléndez, M. L. Álvarez, S. Gallego, M. Ortuño and C. Neipp, "Application of the harmonic balance method to a nonlinear oscillator typified by a mass attached to a stretched wire", J. Sound Vib. 302, 1018-1029 (2007).

[21] A. Beléndez, A. Hernández, A. Márquez, T. Beléndez and C. Neipp, "Analytical approximations for the period of a simple pendulum", Eur. J. Phys. 27, 539-551 (2006).

[22] C. W. Lim, B. S. Wu and W. P. Sun, "Higher accuracy analytical approximations to the Duffing-harmonic oscillator", J. Sound Vib. 296, 1039-1045 (2006).

[23] B. S. Wu, W. P. Sun and C. W. Lim, "An analytical approximate technique for a class of strongly non-linear oscillators”, Int. J. Non-Linear Mech. 41, 766-774 (2006). 
[24] W. P. Sun, B. S. Wu and C. W. Lim, "Approximate analytical solutions for oscillation of a mass attached to a stretched elastic wire", J. Sound Vib. 300, 1042-1047 (2007).

[25] J. H. He, "An elementary introduction to recently developed asymptotic methods and nanomechanics in textile engineering", Int. J. Mod. Phys. B 22, 3487-3578 (2008).

[26] R. E. Mickens, "A generalization method of harmonic-balance”, J. Sound Vib. 111, 515 (1986).

[27] R. E. Mickens and D. Semwogerere, "Fourier analysis of a rational harmonic balance approximation for periodic solutions", J. Sound Vib. 195, 528-530 (1996).

[28] A. Beléndez, E. Gimeno, E. Fernández, D. I. Méndez and M. L. Álvarez, "Accurate approximate solution to nonlinear oscillators in which the restoring force is inversely proportional to the dependent variable", Phys. Scr. 77, 065004 (2008). 